\title{
Artigo/Article
}

\section{Características e tendência da AIDS entre idosos no Estado do Espírito Santo}

\author{
Features and trend of AIDS, among the elderly in the State of Espirito Santo
}

\author{
Lidiane da Silveira Gouvea Toledo ${ }^{1}$, Ethel Leonor Noia Maciel ${ }^{2,3}$, Lívia Carla de Melo Rodrigues ${ }^{1}$, \\ Ricardo Tristão-Sá ${ }^{4}$ e Geisa Fregona ${ }^{1,3}$
}

\begin{abstract}
RESUMO
Introdução: A AIDS entre adultos mais velhos é um problema de saúde pública emergente. Comparamos o perfil epidemiológico e sociodemográfico, bem como a evolução e a tendência da epidemia entre homens e mulheres nas faixas etárias de 50 anos e mais e 20 a 39 anos acometidos pela AIDS, no Estado do Espírito Santo, Brasil. Métodos: Realizamos um estudo de série temporal, com dados secundários do SINAN/AIDS, no período de Janeiro de 1991 a dezembro 2006. Resultados: Neste período, foram notificados 3.382 casos de AIDS em indivíduos com idade entre 20 e 39 anos e 551 casos entre indivíduos com 50 anos e mais. Em ambas as faixas etárias, os mais acometidos são os homens. Há diferenças referentes à raça/ cor, em que a maioria dos mais velhos são brancos $(45,3 \%$ - p-valor $=0,044)$ e os mais jovens pardos $(44,7 \%$, p-valor $=0,003)$. $\mathrm{O}$ analfabetismo prevalece entre os mais velhos $(17,7 \%$ $\mathrm{p}$-valor $=0,001)$. Mais da metade $(80 \%)$ das notificações ocorreu em municípios de médio a grande porte. A principal categoria de exposição foi a heterossexual, em ambos as faixas etárias, com maior frequência para o grupo de 50 anos ou mais $(77,3 \%-p=0,0001)$. A incidência acumulada é maior para a faixa etária de 20 a 39 anos $\left(R^{2}=0,68\right)$; porém, vem aumentando proporcionalmente, entre as duas faixas no decorrer dos anos, com tendência de crescimento significativa para ambas $(p<0,01)$. Conclusões: A epidemia de AIDS pode ser considerada em expansão entre mais velhos no Espirito Santo.
\end{abstract}

Palavras-chaves: Saúde pública. Epidemiologia. Envelhecimento. AIDS.

\section{ABSTRACT}

Introduction: AIDS among older adults is a public health problem emerging. This study compared the demographic and epidemiological profile and the evolution and trend of the epidemic among men and women aged 50 years and older and 20 to 39 years affected by AIDS in the State of Espirito Santo, Brazil. Methods: We conducted a time serie study with secondary data from SINAN/AIDS for the period January 1991 to December 2006. Results: In this period were 3,382 reported cases of AIDS in individuals aged 20 to 39 years and 551 cases among individuals with 50 years or older. In both age groups most affected are men. There are differences related to race or color, where the majority of the older is white $(45.3 \%$ - p-value $=$ $0.044)$ and the young brown $(44.7 \%, \mathrm{p}=0.003)$. Illiteracy prevails among the older $(17.7 \%$ - $p$-value $=0.001)$. More than half $(80 \%)$ of the notifications occurred in cities of medium to large. The main risk factor was heterosexual in both the age groups more frequently to the group of 50 or more $(77.3 \%$ - $\mathrm{p}=0.0001)$. The cumulative incidence is higher for the age group 20 to 39 years $\left(R^{2}=0.68\right)$, but is increasing proportionally between the two bands over the years, with a significant upward trend for both $(\mathrm{p}<0.01)$. Conclusions: The AIDS epidemic among the elderly can be seen growing among older on the Espirito Santo State.

Key-words: Public health. Epidemiology. Aging. AIDS.

1. Faculdade Novo Milênio. Vila Velha, ES. 2. Programa de Pós-Graduação em Saúde Coletiva, Universidade Federal do Espírito Santo. Vitória, ES. 3. Núcleo de Doenças Infecciosas, Universidade Federal do Espírito Santo. Vitória, ES. 4. Escola de Medicina da Santa Casa de Misericórdia. Rio de Janeiro, RJ.

Endereço para correspondência: Dra Ethel Leonor Noia Maciel. Programa de Pós-Graduação em Saúde Coletiva/NDI/UFES. Av. Marechal Campos 1468, Maruípe, 29040-091 Vitória, ES.

e-mail: emaciel@ndi.ufes.br

Recebido para publicação em 19/11/2009

Aceito em 20/01/2010

\section{INTRODUÇÃO}

O significativo aumento no número de casos de AIDS, entre adultos mais velhos, tem sido relatado no mundo inteiro. AUNAIDS e a Organização Mundial de Saúde (OMS) estimam que das 40 milhões de pessoas vivendo com HIV/AIDS no mundo, aproximadamente 2,8 milhões estão na faixa etária igual ou superior a 50 $\operatorname{anos}^{1}$. No Brasil, a taxa de incidência para esta faixa de idade foi de 15,7 por 100.000 habitantes, no ano de 2006, e vem apresentando tendência de crescimento em todas as regiões do país ${ }^{2}$.

Atualmente, passamos por uma revolução no processo de envelhecimento, com novos significados e perspectivas para homens e mulheres, e nesse contexto a sexualidade permanece, apoiada nas tecnologias para tal. A preocupação aqui, se deve ao fato, de que essas pessoas não foram orientadas quando jovens, sobre o uso do preservativo e não se vêem vulneráveis à infecção ${ }^{3}$. Outro fator importante para o aumento de casos foi a introdução da terapia antirretroviral de alta potência (HAART), em meados da década de 90, que aumentou significativamente a sobrevida desses pacientes ${ }^{1,4}$.

Devido à baixa percepção de vulnerabilidade pelos mais velhos, temos por consequência, a pequena demanda pelo teste anti-HIV, ocasionando subnotificação e indeterminação das taxas de infecção para este grupo, torna-as menos precisas, quando comparada a grupos mais jovens ${ }^{5}$.

Outro problema grave é conduzido por muitos profissionais de saúde, que, desconsiderando os mais velhos como sexualmente ativos, deixam escapar oportunidades de detecção precoce do diagnóstico ${ }^{3}$. Em outros aspectos o atraso no diagnóstico, também é influenciado pelas manifestações clínicas da infecção em si, que são confundidas com manifestações de doenças típicas nos pacientes desta faixa etária como, por exemplo, a fadiga e a perda ponderal, que incide em ambos os casos ${ }^{6}$.

É sabido que os pacientes nesta faixa etária apresentam progressão para a imunodepressão de 
forma mais acelerada, observando-se, ainda, maior aparecimento de doenças oportunistas, acrescido de maior probabilidade do advento de outras co-morbidades, o que contribui para o agravamento das condições de saúde $e^{4,7-10}$.

Diante deste quadro, esta pesquisa teve por finalidade analisar, no Espírito Santo, o perfil epidemiológico, socioeconômico e demográfico de indivíduos com AIDS, com idade igual ou superior a 50 anos, e a tendência da AIDS, nas faixas etárias de 20 a 39 anos, além daqueles com idade igual ou superior a 50 anos.

\section{MÉTODOS}

Trata-se de estudo de série temporal realizado com dados secundários provenientes do Sistema Nacional de Notificação de Agravos (SINAN) para AIDS em pacientes adultos da Secretaria de Saúde do Estado do Espírito Santo. As informações foram coletadas no mês de setembro de 2008 e correspondem ao período de 1991 a 2006.

O universo do estudo foi composto por homens e mulheres, notificados como casos de AIDS, divididos em dois estratos etários: de 20 a 39 anos e com idade igual ou superior 50 anos, residentes no Estado do Espírito Santo situado, região Sudeste do Brasil, com extensão territorial de $46.184 \mathrm{~km}^{2}$. Possui 78 municípios, população de 3.352.024 habitantes e densidade demográfica de 72,6 habitantes/ $\mathrm{km}^{2,11}$.

\section{Descrição das variáveis de estudo}

Foram observadas as seguintes variáveis: sexo, raça/cor, escolaridade, município de residência, antecedentes sexuais, uso de drogas, se portadores de hemofilia, história de transfusão sanguínea e hemoderivados.

\section{Análise dos dados}

O cálculo das frequências absoluta e relativa, assim como o cálculo de incidência foram realizados utilizando-se o programa Excel, versão 7.0. Os testes qui-quadrado ou teste exato de Fisher quando necessário, bem como as equações de tendência linear e as estatísticas de ajuste de modelo de regressão linear (valor de $\mathrm{R}^{2}$ e o valor de $\mathrm{p}$ do teste $\mathrm{F}$ de adequação do modelo) foram obtidas com o software estatístico Statistical Package for the Social Sciences, versão 15.0 (SPSS Inc, Chicago, IL, EUA). Em todos os testes, o nível de significância adotado foi de $5 \%$.

Para o cálculo da incidência, utilizou-se a população de referência do senso do ano de 2000 ajustada pelo método direto ${ }^{12}$.

\section{Ética}

Este estudo foi aprovado pelo Comitê de Ética em Pesquisa (CEP) do Centro de Ciências da Saúde da Universidade Federal do Espírito Santo (CCS/UFES), de acordo com a Resolução 196/96, em 28/05/2008

\section{RESULTADOS}

Foram notificados à Coordenação Estadual de DST/AIDS do Espírito Santo, no período de 1991 a 2006, 3.382 casos de AIDS em indivíduos com idades entre 20 e 39 anos e 551 casos entre indivíduos com idade igual ou superior a 50 anos.

Em relação ao gênero, observou-se predomínio do sexo masculino em ambos os grupos. Para a variável raça/cor, os resultados apontam maior número de brancos, entre os mais velhos (139/45,3\%; p-valor $=0,044)$ e maior número de pardos entre os mais jovens (684/44,7\%; p-valor =0,003), como mostra a Tabela 1.

Com relação à escolaridade, houve predomínio de analfabetismo entre os mais velhos )75/17,7\%; p-valor $=0,001$ ) e maior nível de escolaridade entre os mais jovens, demonstrado em todas as categorias desta variável, diferença estatisticamente significativa, exceto na categoria " 12 ou mais anos de estudo", na qual não houve diferença significativa $(198 / 7,49, \mathrm{p}=0,168)$ (Tabela 1$)$.

TABELA 1 - Distribuição dos casos de AIDS entre adultos mais jovens (20-39) e adultos mais velhos ( 50 anos e mais) no Estado do Espírito Santo, segundo variáveis sociodemográficas. 1991- 2006.

\begin{tabular}{|c|c|c|c|c|c|}
\hline \multirow[b]{3}{*}{ Variáveis } & \multicolumn{4}{|c|}{ Faixa etária } & \multirow[b]{3}{*}{ P-valor } \\
\hline & \multicolumn{2}{|c|}{$20-39$} & \multicolumn{2}{|c|}{$\geq 50$} & \\
\hline & $\mathrm{n}^{\mathrm{o}}$ & $\%$ & $\mathrm{n}^{\mathrm{o}}$ & $\%$ & \\
\hline \multicolumn{6}{|l|}{ Gênero } \\
\hline masculino & 2.111 & 62,4 & 338 & 61,4 & - \\
\hline feminino & 1.271 & 37,6 & 213 & 38,7 & 0,62 \\
\hline \multicolumn{6}{|l|}{ Raça/cor* } \\
\hline branca & 599 & 39,1 & 139 & 45,3 & 0,044 \\
\hline preta & 219 & 14,3 & 55 & 17,9 & 0,105 \\
\hline amarela & 27 & 1,76 & 4 & 1,3 & 0,807 \\
\hline parda & 684 & 44,7 & 109 & 35,5 & 0,003 \\
\hline indígena & 2 & 0,14 & 0 & 0 & - \\
\hline total & 1531 & 100 & 307 & 100 & \\
\hline ignorado & 1.851 & 54,7 & 244 & 44,3 & 0,001 \\
\hline \multicolumn{6}{|c|}{ Anos de Estudo* } \\
\hline nenhum & 147 & 5,56 & 75 & 17,7 & 0,001 \\
\hline de 1 a 3 & 501 & 18,9 & 99 & 23,3 & 0,035 \\
\hline de 4 a 7 & 1.090 & 41,2 & 136 & 32 & 0,0003 \\
\hline de 8 a 11 & 709 & 26,8 & 75 & 17,7 & 0,001 \\
\hline$\geq 12$ & 198 & 7,49 & 40 & 9,41 & 0,168 \\
\hline total & 2645 & 100 & 551 & 100 & - \\
\hline ignorado & 737 & 21,8 & 126 & 22,9 & 0,79 \\
\hline
\end{tabular}

* Calculado apenas os valores válidos, excetuando os ignorados.

Quanto à distribuição dos casos de AIDS, nos municípios capixabas, não houve diferenças significativas quando comparamos os diferentes grupos etários em municípios com população de até 50 mil habitantes ou mais ( $p$-valor $=0,257$ ), como se pode ver na Tabela 2. No entanto, constatou-se que dentre os municípios com mais de 50 mil habitantes, os que compõem a região metropolitana da grande Vitória foram responsáveis por, aproximadamente, $80 \%$ das notificações, em ambas as faixas etárias.

A categoria de exposição heterossexual mostrou-se mais frequente entre os mais velhos que entre os mais jovens (342/77,3\%;

TABELA 2 - Distribuição dos casos de AIDS entre adultos mais jovens (20-39 anos) e adultos mais velhos ( 50 anos e mais) no Estado do Espírito Santo, segundo município de residência. 1991-2006.

\begin{tabular}{|c|c|c|c|c|c|}
\hline \multirow[b]{3}{*}{ Municípios } & \multicolumn{4}{|c|}{ Faixa etária } & \multirow[b]{3}{*}{ P-valor } \\
\hline & \multicolumn{2}{|c|}{$20-39$} & \multicolumn{2}{|c|}{$\geq 50$} & \\
\hline & $\mathrm{n}^{\mathrm{o}}$ & $\%$ & $\mathrm{n}^{\mathrm{o}}$ & $\%$ & \\
\hline Até 50.000 mil hab. & 444 & 13,3 & 82 & 15 & \multirow{4}{*}{0,257} \\
\hline Maior que 50.000 mil hab. & 2887 & 86,7 & 460 & 85 & \\
\hline Não preenchido & 1 & 0,03 & 0 & 0 & \\
\hline Não classificado & 50 & 1,5 & 9 & 1,7 & \\
\hline
\end{tabular}


p-valor $=0,0001)$. De modo contrário, a categoria homossexual, foi maior entre os mais jovens, quando comparada com a população mais velha $(487 / 16,9 \%$, p-valor $=0,0001)$; bem como o uso de drogas injetáveis (128/4,4\%; p-valor=0,001) (Tabela 3).

Quanto à evolução da epidemia, nos últimos anos, observa-se na Figura 1, que a incidência acumulada é maior na faixa etária de 20 a 39 anos (equação de regressão: $-4546,65+2,29 *$ ano; $R^{2}=0,68$ ) que na faixa de maior que 50 (equação de regressão: $-1056,57+0,53^{*}$ ano; $\left.\mathrm{R}^{2}=0,71\right)$; porém, vem aumentando proporcionalmente entre as duas faixas no decorrer dos anos com pico no ano de 2005. A tendência de crescimento foi significativa para as duas faixas etárias $(p<0,01)$.

TABELA 3 - Distribuição dos casos de AIDS entre adultos mais jovens (20-39) e adultos mais velhos ( 50 anos e mais) no Estado do Espírito Santo, segundo categoria de Exposição. 1991-2006.

\begin{tabular}{|c|c|c|c|c|c|}
\hline & \multicolumn{4}{|c|}{ Faixa etária } & \multirow[b]{3}{*}{ p-valor } \\
\hline & \multicolumn{2}{|c|}{$20-39$} & \multicolumn{2}{|c|}{$\geq 50$} & \\
\hline & $\mathrm{n}^{\mathrm{o}}$ & $\%$ & $\mathrm{n}$ o & $\%$ & \\
\hline \multicolumn{6}{|l|}{ Categoria de Exposição } \\
\hline heterossexual & 1.882 & 65,7 & 342 & 77,3 & 0,0001 \\
\hline homossexual & 487 & 16,9 & 37 & 8,4 & 0,0001 \\
\hline bissexual & 340 & 11,8 & 55 & 12,4 & 0,72 \\
\hline uso de drogas injetáveis & 128 & 4,4 & 2 & 0,45 & 0,001 \\
\hline \multirow[t]{2}{*}{ hemofilia } & 10 & 0,35 & 1 & 0,23 & 1,00 \\
\hline & 11 & 0,38 & 5 & 1,13 & 0,052 \\
\hline \multicolumn{6}{|c|}{ Transfusão de sangue/hemoderivados } \\
\hline perinatal & 12 & & 0 & 0 & - \\
\hline total $^{*}$ & 2870 & 100 & 442 & 100 & - \\
\hline não classificados & 1 & & 0 & & - \\
\hline ignorados & 551 & & 109 & & 0,031 \\
\hline Totalgeral ${ }^{* *}$ & 3.422 & 100 & 551 & 100 & \\
\hline
\end{tabular}

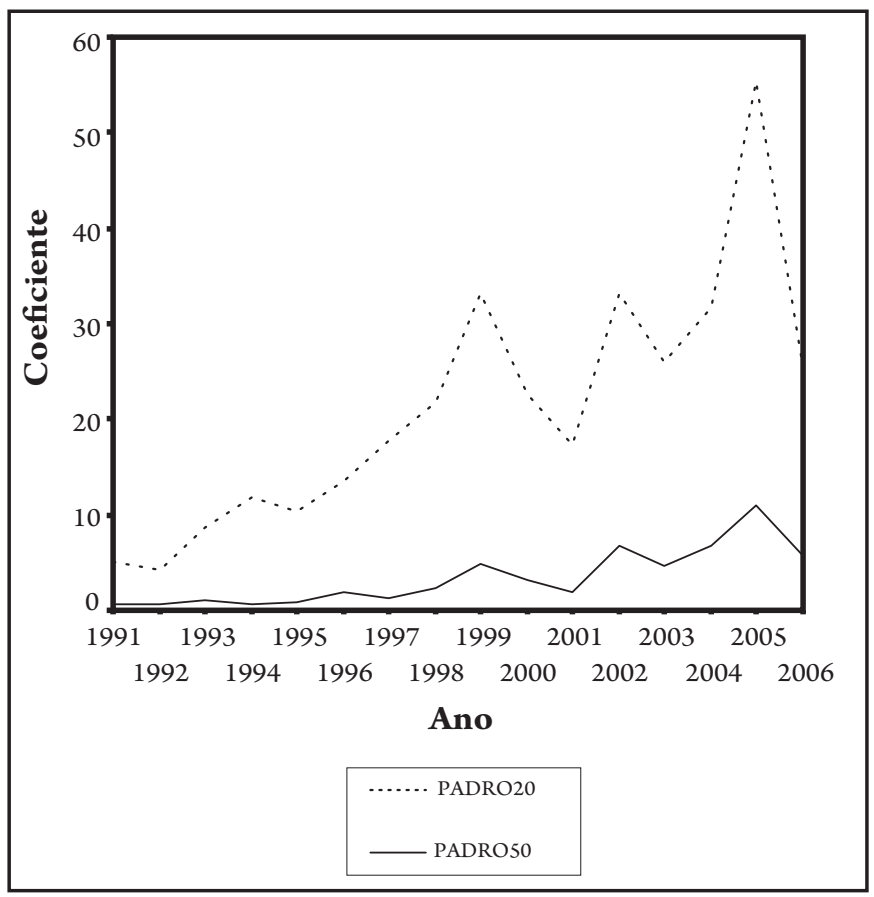

FIGURA 1 - Tendência de AIDS na faixa etária de 20 a 39 anos e 50 e mais, no Estado do Espírito Santo. 1991-2006.

\section{DISCUSSÃO}

Neste estudo, identificamos maior proporção do número de casos de AIDS na faixa etária de 20 a 39 anos e pertencentes ao sexo masculino. Desde o começo da epidemia no Brasil, o grupo etário mais atingido, em ambos os sexos, tem sido o de 20 a 39 anos perfazendo cerca de $70 \%$ do total de casos até junho de $2000^{13,14}$.

$\mathrm{Na}$ categoria raça/cor, os mais atingidos pela AIDS na faixa etária de 20 a 39 anos foram os de cor parda, o que corrobora com estudo anterior realizado no Brasil que demonstrou aumento de AIDS entre pessoas categorizadas como pardas ${ }^{15}$. Ainda, dois estudos norte americanos, também, demonstraram crescimento no número de casos na população não branca ${ }^{16,17}$. Uma possível explicação apontada por estes autores seria a pauperização da doença contribuindo para isto a dificuldade no acesso aos serviços de saúde. Chama a atenção, o número de dados ignorados na categoria raça/cor tendo como uma das possíveis explicações o fato de ter sido inserida no SINAN somente a partir de $2001^{18}$.

Quanto à escolaridade, considerado o melhor indicador para mensurar o nível sócio-econômico associado à saúde da população ${ }^{19,20}$ prevaleceu o nível educacional de 4 a 7 anos em ambas as faixas etárias corroborando com dados encontrados em outro estudo brasileiro ${ }^{21}$. Resultados semelhantes foram demonstrados por Fonseca cols ${ }^{14}$ que encontraram uma proporção de $69,4 \%$ entre aqueles que tinham cursado até o $1^{\circ}$ grau completo (constituído por indivíduos desde analfabetos até ensino fundamental completo), o que está de acordo com nosso estudo.

Podemos perceber neste estudo que a proporção de casos se concentra em municípios de médio a grande porte, com mais de 50 mil habitantes. A região metropolitana da grande Vitória abriga quase a metade da população total do Espírito Santo e corresponde a $57 \%$ da população urbana do estado ${ }^{22}$.

Estudo realizado em Recife em 2007, com todas as idades, mostrou que a concentração dos casos de AIDS se deu na região metropolitana com $77,26 \%$ dos $\operatorname{casos}^{23}$, resultados semelhantes aos nossos. Contudo, há que se considerar se os resultados encontrados nos municípios do interior são realmente a dos dados apresentados ou se existiriam casos subnotificados, supondo-se que a população nessas áreas de modo geral é mais conservadora e, o preconceito é, ainda, uma barreira considerável para a realização do teste anti-HIV, o que possibilitaria uma maior procura em centros urbanos para efetivarem o diagnóstico e tratamento.

Em relação à categoria de exposição, a via heterossexual foi a mais expressiva nas duas faixas etárias confirmando que a prática sexual sem proteção adequada se constitui como a mais importante via de transmissão também entre os mais velhos. A relevante transmissão heterossexual em pessoas mais velhas pode ter como fatores predisponentes a resistência ao uso de preservativo e o advento de grande número de drogas farmacológicas facilitadoras da relação sexual, podendo influenciar as práticas homossexuais igualmente desprotegidas ${ }^{24}$.

Ainda em relação à categoria de exposição, mesmo que em pequeno número, chama a atenção o uso de drogas injetáveis entre os adultos mais velhos, grupo este que raramente é considerado tóxicodependente. Os profissionais de saúde tendem a não investigar o uso de drogas nestes pacientes, assumindo erroneamente que este grupo 
não é passível de tal comportamento ou se o foi no passado, não se constitui fato relevante para o presente, no momento da consulta ${ }^{12,25}$. É pequeno o percentual de transmissão do HIV por drogas injetáveis em ambos os grupos, no entanto deve ser considerado como fator importante na investigação epidemiológica do $\mathrm{caso}^{26}$.

Em termos de incidência, observou-se tendência de crescimento significativa em ambas as faixas etárias. Ao analisar o comportamento da incidência de AIDS no grupo etário de 50 anos e mais entre 1990 a 2003, no Brasil e suas macrorregiões, Sousa, Silva e Montarroyos ${ }^{27}$ identificaram tendência crescente e estatisticamente significante em todas as regiões, sendo maiores no Sudeste corroborando com os achado deste estudo.

É importante ressaltar que a principal limitação do estudo foi a natureza dos dados secundários. Problemas inerentes à confiabilidade da informação seja por erros decorrentes de digitação e registro, e a própria cobertura da informação impõem restrições quanto à validade dos dados apresentados ${ }^{25}$. Entretanto, este problema só será transposto na medida em que a vigilância dos dados for efetivada em todos os níveis da atenção a saúde, principalmente, no que concernem às doenças infecciosas. A confiabilidade, completude e atualização dos dados podem melhorar a sua qualidade e privilegiará a tomada de decisões, mas, para tanto, é necessário que tanto o nível municipal quanto o estadual identifiquem fatores que possam interferir na adequada atualização dos dados referentes à notificação e ao acompanhamento dos casos, investindo na melhoria da qualidade da informação no estado do Espírito Santo ${ }^{28}$.

Por fim, fazem-se necessárias ações integradas nos serviços de saúde para o conhecimento da magnitude da doença e para a vigilância no seu controle ${ }^{29}$. Ações de educação permanente para profissionais de saúde na assistência à saúde dos mais velhos poderiam refletir na diminuição de erros e demora no diagnóstico e ainda, na subnotificação de casos $^{3}$. Além disso, o desenvolvimento de estratégias de prevenção que possibilitem maior conhecimento da população acima de 50 anos acerca da sua vulnerabilidade tem sido recomendado por órgãos governamentais e devem ser incorporadas à rotina dos serviços ${ }^{23}$.

\section{AGRADECIMENTOS}

Agradecemos a secretária estadual de DST/AIDS do Estado do Espírito Santo, a Dra Sandra Fagundes Moreira-Silva e a Dra Regina Lúcia Ribeiro Alves, pelo fornecimento dos dados.

\section{CONFLITO DE INTERESSE}

Os autores declaram não haver nenhum tipo de conflito de interesse no desenvolvimento do estudo.

\section{SUPORTE FINANCEIRO}

Fundação de Apoio à Ciência e Tecnologia do Espírito Santo FAPES/FUNCITEC e ICOHRTA 5 U2R TW006883-02.

\section{REFERÊNCIAS}

1. Joint United Nations Programme on HIV/AIDS; World Health Organization AIDS epidemic update. $\mathrm{n}^{\circ}$ 8, August, 2008.

2. Ministério da Saúde. Secretaria de Vigilância em Saúde. Programa Nacional de DST e AIDS. Boletim epidemiológico AIDS e DST, ano V, nº 1, 01a , 26a , semanas epidemiológicas, jan a jun de 2008.
3. Inelmen EM, Gasparini G, Enzi G. HIV/AIDS in older adults. A case report and literature review. Geriatrics 2005; 60:26-30.

4. Nguyen N, Holodniy M. HIV infection in the elderly. Clin Interv Aging 2008; 3:453-472.

5. Mack KA, Ory MG. AIDS and Older Americans at the End of the Twentieth Century. J Acquir Immune Defic Syndr Hum Retrovirol 2003; 33 (suppl 2):68-75.

6. Siegel K, Dean L, Schrimshaw E. Symptom ambiguity among late-middle aged and older adults with HIV. Res Aging 1999; 21:595-618.

7. Adler WH, Nagel JE. Acquired immunodeficiency syndrome in the elderly. Drugs Aging 1994; 5:410-416.

8. El-Sadr W, Gettler J. Unrecognized human immunodeficiency virus infection in the elderly. Arch Intern Med 1995; 2:184-186.

9. Wallace JI, Paauw DS, Spach DH. HIV infection in older patients: When to suspect the unexpected. Geriatrics 1993; 6:61-70.

10. Boudes P. HIV infection in the elderly. Compr Ther 1991; 9:39-42.

11. Programa das Nações Unidas Para o Desenvolvimento (PNUD). 2004. Atlas do Desenvolvimento Humano no Brasil. Disponível em: <http://www.pnud.org. br/atlas/. (Acesso em: 28 nov. 2008).

12. Gordis L. Measuring the occurrence of disease: II. Mortality. In: Gordis L, editor Epidemiology. $3^{\text {rd }}$. Philadelphia: Elsevier Saunders 2004. p. 48-70.

13. Brito AM, Castilho EA, Szwarcwald CL. AIDS e infecção pelo HIV no Brasil: uma epidemia multifacetada. Rev Soc Bras Med Trop 2001; 34:207-217.

14. Fonseca MGP, Szwarcwald CL, Bastos FI. Análise sociodemográfica da epidemia de Aids no Brasil, 1989-1997. Rev Saúde Pública 2002; 6:678-685.

15. Fry PH, Monteiro S, Maio MC, Bastos FI, Santos RV. AIDS tem cor ou raça? Interpretação de dados e formulação de políticas de saúde no Brasil. Cad Saude Publica 2007; 3:497-523.

16. Mack KA, Bland SD. HIV Testing Behaviors and Attitudes Regarding HIV/AIDS of Adults Aged 50-64. Gerontologist 1999; 6: 687-694.

17. Linley L, Hall HI, An Q, Wheeler W. HIV/AIDS diagnoses among persons fifty years and older. In 33 states, 2001-2005. National HIV Prevention Conference, Abstract. Atlanta. December. 2007; B08-1.

18. Espírito Santo. Secretaria de Estado da Saúde. Coordenação Estadual de DST/ AIDS. Boletim epidemiológico, Até dezembro de 2007; n²0.

19. Kunst AE, Mackenbach JP. The size of mortality differences associated with educational level in nine industrialized countries. Am J Public Health 1994; 84:932-937.

20. Sorlie PD, Backlund E, Keller JB. US mortality by economic, demographic, and social characteristics: The National Longitudinal Mortality Study. Am J Public Health 1995; 85:949-956.

21. Ferreira RCSL, Dias JO, Mello RS, Sakae TM. Perfil Epidemiológico da Síndrome da Imunodeficiência: Adquirida na região da Associação de Municípios da Região de Laguna (AMUREL) de 1987 a 2006. Arq Catarinenses de Medicina 2008; 37:19-24.

22. Instituto Brasileiro de Geografia e Estatística (IBGE). Estimativas para Contagem da População 2007. 2007. Disponível em: < www.ibge.gov.br (Acesso em: 01 de julho 2009).

23. Ventura AACM, Ventura CCM, Ávila MP, Sposito A. Características dos pacientes HIV positivos do estado de Pernambuco nos últimos 20 anos. An Fac Med Univ Fed Pernamb 2007; 2:122-126.

24. Lindau ST, Schumm LP, Laumann EO, Levinson W, O’Muircheartaigh CA, Waite LJ.A study of sexuality and health among older adults in the United States. N Engl J Med 2007; 357:762-774.

25. Pottes FA, Brito AM, Gouveia GC, Araújo EC, Carneiro RM. Aids e envelhecimento: características dos casos com idade igual ou maior que 50 anos em Pernambuco, de 1990 a 2000. Rev Bras Epidemiol 2007; 10:338-351.

26. Linsk NL. HIV among older adults. AIDS Reader 2000; 10:430-440.

27. Sousa JL, Silva MDP, Montarroyos UR. Tendência de AIDS no grupo etário de 50 anos e mais no período anterior e posterior à introdução de medicamentos para disfunção erétil: Brasil, 1990 a 2003. Rev Bras Geriatria e Gerontol 2007; 10:203-216.

28. Doyle TJ, Glynn MK, Groseclose SL. Completeness of notifiable infectious disease reporting in the United States: an analytical literature review. Am J Epidemiol 2002; 155:866-874.

29. Araújo VL B, Brito DMS, Gimeniz MT, Queiroz TA, Tavares CM. Características da Aids na terceira idade em um hospital de referência do Estado do Ceará, Brasil. Rev Bras Epidemiol 2007; 10:544-554. 